\title{
" Je meesmes me sui mis en queste, et assez ai traveillé » : Questes, quinze ans de recherche et d'amitié
}

Florian Besson, Adrien Carbonnet, Sarah Delale, Pauline Guena et Elodie Pinel

\section{(2) OpenEdition}

1 Journals

Édition électronique

URL : http://journals.openedition.org/questes/4357

DOI : 10.4000/questes.4357

ISSN : 2109-9472

Éditeur

Les Amis de Questes

\section{Édition imprimée}

Date de publication : 15 juin 2016

ISSN : 2102-7188

Référence électronique

Florian Besson, Adrien Carbonnet, Sarah Delale, Pauline Guena et Elodie Pinel, « « Je meesmes me sui mis en queste, et assez ai traveillé »: Questes, quinze ans de recherche et d'amitié », Questes [En ligne], Numéro spécial | 2016, mis en ligne le 20 juin 2016, consulté le 20 avril 2019. URL : http:// journals.openedition.org/questes/4357; DOI : 10.4000/questes.4357

Ce document a été généré automatiquement le 20 avril 2019

(C) Association des amis de «Questes » 


\title{
" Je meesmes me sui mis en queste, et assez ai traveillé »: Questes, quinze ans de recherche et d'amitié
}

\author{
Florian Besson, Adrien Carbonnet, Sarah Delale, Pauline Guena et Elodie \\ Pinel
}

\section{NOTE DE L'AUTEUR}

La citation du titre est tirée du Roman de Tristan en prose, éd. Renée L. Curtis, Cambridge, D. S. Brewer, 1985, t. III, troisième partie, paragraphe 788, p. 98.

1 En 2001, dans le prolongement d'un séminaire d'élève de l'ENS, un groupe de doctorants se rassemble sur l'impulsion d'Estelle Doudet. Il prend le nom de « Questes » :

\begin{abstract}
Nous aurions pu nous passer de nom ou prendre un intitulé plus universitairement correct comme " groupe de travail sur le Moyen Âge ». Mais nous trouvions qu'un nom original, éventuellement déclinable en substantif pour nous désigner, nous donnerait une forme d'identité. D'où l'idée d'un mot médiéval, soit en latin soit en ancien français puisque nous étions en France. Nous avons pensé à diverses possibilités, autour de l'idée de recherche et de cheminement en commun : je crois me souvenir de «trivium» par exemple. Andrea Martignoni, qui avait eu tout de suite envie de construire un site internet, a attiré notre attention sur l'importance d'un nom qui puisse devenir aussi celui d'un domaine. Nous sommes tombés d'accord sur «Questes». Au pluriel bien sûr, pour souligner notre diversité. Nous avons aussi décidé que nous prononcerions le $-s$ central pour marquer notre identité à l'oral. Être les questeurs nous a vraiment unis : nous pouvions désormais parler de nous en nous désignant par un nom à la fois facile à retenir et plaisamment évocateur (errance chevaleresque ou Saint Graal de la thèse ?) ${ }^{1}$.
\end{abstract}

En 2016, Questes existe toujours et a considérablement évolué : en même temps que ses pratiques ont changé, sa reconnaissance institutionnelle a fortement augmenté. Cet article est avant tout l'occasion de redire toute notre gratitude envers ces institutions qui n'ont cessé de nous soutenir, humainement et financièrement : l'Ecole Doctorale 1 de 
l'Université Paris-Sorbonne, l'UMR 8596 Roland Mousnier, l'EA 4349 de l'Université ParisSorbonne, l'Institut Universitaire de France, le Centre des Sciences de la Littérature Française de l'Université Paris Ouest Nanterre La Défense et l'Univeristé Grenoble Alpes. Pour ses quinze ans d'existence, nous voudrions en ces lignes rendre hommage aux générations successives des Questeurs qui travaillèrent à tisser des liens étroits entre les chercheurs médiévistes.

\section{Du groupe à l'association}

3 S'organisant d'abord autour de courtes interventions de cinq à dix minutes et de format très libre (compte rendu d'un ouvrage, réflexion autour d'une citation, d'un thème...) et laissant une large place à la discussion, les séances de Questes ont durant les premières années décliné des thèmes choisis par les membres les plus actifs. Aucun responsable de thème n'étant porteur de projet, charge revenait au secrétaire du groupe de lancer la discussion et de rédiger le compte rendu de séance, lequel était mis en ligne. La liste de diffusion, très active, relayait plus d'une annonce de parution ou de manifestation scientifique par jour et comptait plus de deux cents inscrits ${ }^{2}$. Cette activité de veille scientifique ouvrait d'ailleurs chaque séance, où les dernières parutions remarquables étaient signalées par Pierre Levron ou Andrea Martignoni. L'année 2003 vit se tenir la première journée d'étude ; cette forme de rencontre scientifique sera reconduite, d'abord tous les trois ans puis tous les deux ans.

4 Bénéficiant d'une dotation budgétaire stable dès ses débuts grâce au soutien de l'équipe dirigée par Jacqueline Cerquiglini-Toulet, le groupe travaille très tôt avec le concours de la reprographie de Paris IV. Cela lui permet d'envoyer, sur l'idée de Nelly Labère, Andrea Martignoni et Silvère Menegaldo, un bulletin papier aux professeurs soutenant le groupe ${ }^{3}$ , avant que ce lectorat ne soit élargi à d'autres maitres de conférences et professeurs en littérature et en histoire médiévales. Outre le compte-rendu des séances, les premiers numéros présentent un résumé des communications présentées dans le séminaire, résumé qui prend de plus en plus d'ampleur au fil des années ${ }^{4}$. Jacqueline CerquigliniToulet fait inscrire les actions du groupe dans le rapport d'activité de l'UMR et concourt à faire publier les actes des journées d'étude aux PUPS. Grâce à ce soutien, et sur suggestion de Françoise Mélonio, Questes est reconnu comme un des premiers groupes de doctorants en sciences humaines et sociales en 2003.

Devenu association de loi 1901, au moment du changement de statut du centre d'« Étude et édition de textes médiévaux » d'UMR en équipe d'accueil, Questes s'institutionnalise en adoptant un format de parole plus encadré: les thèmes donnent lieu à des communications plus fournies, qui sont publiées sous forme d'articles au sein du bulletin. Un travail proprement éditorial est alors requis et la nécessité de mettre le bulletin sous la responsabilité de coordinateurs du thème s'impose: des normes éditoriales sont élaborées, des responsables du bulletin sont nommés, les thèmes sélectionnés le sont par vote en début d'année et correspondent à des projets proposés par les membres volontaires. Le bulletin est déposé à la BnF; aujourd'hui, la venue de doctorants pour assister aux séminaires peut donner lieu dans certaines écoles doctorales à la validation de la partie «formation" du doctorat, ou à des financements dans le cas de doctorants résidant en dehors de la région parisienne. 
6 Le site internet a également connu plusieurs versions : le site originel a été transféré entre 2012 et 2015 vers des plateformes institutionnelles. Un carnet de recherche, hébergé par la plateforme hypotheses.org d'OpenEdition, regroupe l'ensemble des informations et comptes rendus de l'association. Quant au bulletin, toujours doté d'un tirage papier conséquent, il paraît désormais sur la plateforme revues.org et a été renommé « revue » en lien avec les exigences d'OpenEdition. Le transfert, initié par JeanBaptiste Camps et Diane Chamboduc de Saint-Pulgent, a mobilisé sur son temps libre une équipe de Questeurs durant deux années entières. Lue et reconnue pour son sérieux, la revue est régulièrement citée dans les séminaires de recherche et les publications scientifiques; le vœu des membres fondateurs d'éviter la parole magistrale n'a en cela pas été respecté. Dans la mesure où cette entorse à l'esprit de Questes n'entrave pas l'idéal d'entraide des doctorants, gageons que c'est un moindre mal.

Questes s'est en effet d'emblée inscrit sous le triple signe de l'internationalité, de l'interdisciplinarité et de la convivialité.

Interdisciplinarité, tout d'abord. Pratique usuelle des séminaires d'élèves de l'ENS, le croisement des disciplines se déclina à l'origine par la présence, au sein du groupe, de doctorants associant plusieurs approches d'un même sujet : lettres et musique, histoire de l'art ou histoire. Si le groupe fut d'abord centré sur la recherche en littérature, la proportion croissante de doctorants en histoire médiévale fit bouger les lignes et fait aujourd'hui de Questes un centre de réflexion tant littéraire qu'historique, sans obliger pour autant les littéraires à se borner à une approche socio-critique des textes ni contraindre les historiens à privilégier l'histoire des mentalités. Littéraires et historiens croisent leurs regards et entrent dans un dialogue véritable au cours des deux temps structurant la vie du groupe, les séminaires et les journées d'études.

Internationalité, ensuite. Mus par l'envie de «comprendre les formatages nationaux avant de commencer à faire de la recherche ${ }^{5}$ ", les membres fondateurs avaient en tête le modèle d'une « sodalitas savante ${ }^{6}$ » à la fois professionnelle et amicale.

Convivialité, enfin. Le souvenir le plus marquant des années Questes, pour tous les anciens membres ayant répondu à nos questions, n'est autre que le repas partagé après la séance, "moment d'échange presque aussi important que la séance elle-même ${ }^{7}$ ». Ce consensus n'est pas, nous l'espérons, symptomatique de la propension des doctorants à préférer le réconfort à l'effort, mais plutôt la marque de l'objectif que s'était donné le groupe d'« échanger sur nos textes, thèmes ou archives et les partager " dans une "volonté d'autorité horizontale ». Qu'ils aient été en poste dans un lycée, doctorants en province ou allocataires-moniteurs dans une université parisienne, tous les anciens membres de Questes soulignent que le groupe leur a permis de «briser la solitude du chercheur " ", certains ajoutant même : "je n'aurais peut-être jamais terminé ma thèse sans Questes ${ }^{9}$ ». Si la fréquentation du groupe a permis à tous de sortir de l'isolement, elle a également été pour beaucoup l'occasion de «faire connaissance avec de jeunes chercheurs, dont beaucoup sont devenus des collègues et restés des amis ${ }^{10}$ ». Les projets collectifs, du temps de Questes et au-delà, n'en ont été que facilités, la participation aux séminaires créant « un désir de recherche » chez beaucoup. Et on connaît le lien culturel fort qui unit la conversation au banquet. C'est par un «banquet médiéval de la fin du XIV siècle français » que s'était achevée la première année du séminaire :

Juin 2002 : Réalisation du Banquet : tables de bois et chandelles, cuisine et boissons, musiques et jongleurs, costumes d'époque au rendez-vous sous les frondaisons d'une cour à l'ENS d'Ulm. Clôture de la première année du séminaire ${ }^{11}$. 
11 Le repas qui conclut chaque séance redit en quelque sorte indéfiniment cette relation entre parole et convivium.

Comme Adam nommant les animaux au paradis terrestre, les Questeurs ont pris droit de cité dans le monde à travers leur nom: nom qui dit la recherche, mais qui dit aussi l'aventure. C'est surtout cet esprit qui s'est conservé depuis l'origine. En 2007, le groupe proposait deux sessions au XII ${ }^{e}$ Congrès International de la Société de Littérature Courtoise $^{12}$. En octobre 2016, l'association doit organiser une table-ronde aux Rendezvous de l'histoire de Blois. Un projet de cycle de conférences est en cours d'élaboration avec le musée de Cluny; un partenariat se met en place avec les Jeunes Chercheurs Médiévistes de Genève, qui permettrait aux membres d'aller les saus menus, tel le pèlerin et sa coquille, d'un pays l'autre le temps d'un séminaire. Défis, épreuves, compagnonnages, voyages : tels sont les aventures qui continueront toujours à l'arrièreplan de la revue et du studieux séminaire de l'association.

\section{La revue Questes en quelques chiffres}

Depuis 2002 et son premier numéro, Tout un programme, Questes a publié trente-trois numéros de son bulletin; un peu plus de deux par an, en moyenne. Un résultat, somme toute, assez impressionnant, surtout quand l'on se rappelle que les responsables de thème et les responsables du bulletin, qui tous assurent une relecture dense et poussée des articles, sont tous doctorants ou jeunes docteurs et tous bénévoles.

\section{Des responsables de thème}

En théorie, chaque bulletin est coordonné par deux Questeurs, mais plusieurs l'ont été par une seule personne (14, Violences médiévales; 16 , Secret, public, privé; 22 , La route des pèlerins ; 25, L'habit fait-il le moine ?). Seuls deux numéros ont été coordonnés par trois personnes (24, L'identité, et 29, Copie, authenticité, originalité) : Questes préfère le couple au triumvirat. On dénombre en tout cinquante-cinq coordinateurs pour ces trente-trois numéros. En favorisant systématiquement la codirection, Questes joue un rôle important dans la pratique de la co-écriture - capitale dans les sciences exactes et trop peu utilisée par nos disciplines. Quant à la pluridisciplinarité, c'est l'un des principes fondateurs de Questes, depuis ses débuts: s'y côtoient en effet littéraires, historiens, historiens de l'art, linguistes, musicologues, philosophes et archéologues. Ces rencontres se voient davantage à l'intérieur même des bulletins qu'au niveau des responsables de thème, puisque seuls neuf bulletins ont été codirigés par une équipe pluridisciplinaire ${ }^{13}$. En revanche, les journées d'étude de 2011, 2013 et 2015 ont toutes été organisées par des équipes résolument pluridisciplinaires.

Si les premières années de l'association ont été marquées par une majorité de littéraires, souvent des doctorant(e)s dirigé(e)s par Jacqueline Cerquiglini-Toulet ${ }^{14}$, ces dernières années ont vu s'investir des historiens en nombre croissant, la plupart dirigés par Élisabeth Crouzet-Pavan ${ }^{15}$. La charnière est assez nette entre 2009 et 2010, et cette tendance ne fait que se renforcer à partir de 2013; les numéros 26 (Trouver la paix), 30 ( L'erreur, l'échec, la faute), 32 (Faire communauté) et 33 (Finir le Moyen Âge) sont codirigés par deux doctorants d'Elisabeth Crouzet-Pavan. Ce tournant s'observe également au niveau des articles, qui font une place croissante à l'histoire. 


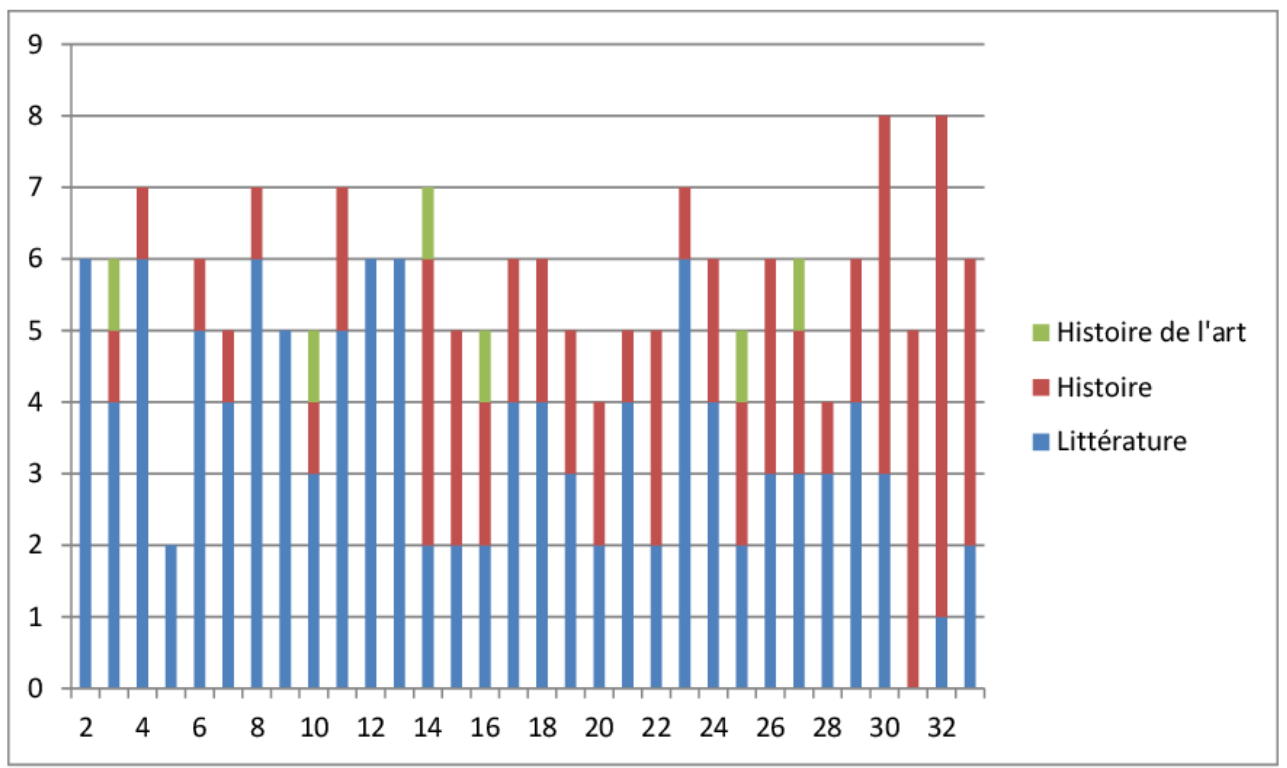

16 Il faut noter que certains Questeurs ont joué, plusieurs fois, un rôle-clé, coordonnant plusieurs numéros successifs du bulletin : en 2006 et 2007, Julien Abed et Sophie Albert dirigent ainsi, seuls ou à eux deux, les numéros 8, 9, 10 et 11 ! Cette activité concentrée souligne le grand investissement de ces auteurs, capables, dans des périodes où l'on imagine les Questeurs peut-être moins nombreux, de tenir l'association à bout de bras et de plume.

On retrouve parfois des coordinateurs à plusieurs années d'écart: Amandine Mussou prend en charge les numéros 13 (Figures royales à l'ombre du mythe) et 18 (La règle du jeu), en 2008 et 2010 ; Céline Ménager le numéro 19 (Les manifestations divines) en 2010 puis le 27 (Naissances) en 2014 ; Magali Cheynet dirige les numéros 22 (La route des pèlerins) en 2011 et 29 (La prophétie) en 2015. Il semble donc que la participation à Questes accompagne et sous-tende la progression de la thèse.

Fréquemment, mais non systématiquement, les responsables de thème ont déjà publié un ou plusieurs articles lorsqu'ils prennent en charge la rédaction d'un numéro. Sans surprise, l'organisation de la journée d'études bisannuelle de Questes est le plus souvent prise en charge par des Questeurs qui figurent parmi les plus actifs du moment, et qui ont, généralement, dirigé un ou plusieurs bulletins auparavant : Estelle Doudet organise la première journée d'études en 2003 (La mort écrite); Sophie Albert s'occupe de la journée d'études de 2005 (Laver, monder, blanchir) ; Laëtitia Tabard et Cécile Becchia prennent en charge celle de 2011 (Intus et foris), et toutes deux co-dirigent un numéro qui sort soit un peu avant (numéro 18, La règle du jeu, 2010), soit juste après (numéro 24, L'identité, 2012). De même, on voit Diane Chamboduc de Saint-Pulgent co-diriger les numéros 20 et 24 ( Maris et femmes, 2011 et Le doute, 2012) et co-organiser la journée d'études de 2013 ( Précarité, instabilité, fragilité). Florian Besson et Julie Pilorget, qui codirigent respectivement le numéro 31 (L'échec, l'erreur, la faute) et le numéro 32 (Faire communauté), issus des séminaires de l'année 2012-2013, co-organisent avec d'autres la journée d'études de 2015 (Créer). 


\section{Des auteurs}

En trente-trois numéros, cent quarante auteurs ont écrit pour Questes. Il est impossible de l'estimer précisément, mais on peut penser qu'assez souvent, cet article est le premier article publié. Questes a ainsi un rôle important, permettant à de jeunes chercheurs - doctorants ou même masterants - de prendre la parole en public pour la première fois dans un contexte amical et détendu.

De nombreux auteurs ont écrit dans plusieurs numéros. Sophie Albert rédige cinq articles entre 2006 et 2008 ; Julien Abed six articles entre 2006 et 2009. La palme revient à Andrea Martignoni, qui ne publie pas moins de seize travaux entre 2002 et 2004:articles, comptes rendus, bibliographies... D'autres publient un article de temps en temps pendant plusieurs années : Mathilde Grodet signe un article en 2004, 2006, 2009 et 2010, et codirige un numéro en 2011 ; Pierre Levron écrit quatorze comptes rendus et articles entre 2002 à 2011. La plupart du temps cependant, les articles sont publiés dans une fourchette de deux à trois ans : la participation à Questes dépend donc étroitement de la conduite de la thèse, et, le plus souvent, de la vie à Paris.

\section{Des thèmes}

Il vaut la peine de citer l'ensemble des thèmes que nous avons traité au cours de ces quinze ans.

\begin{tabular}{|l|l|l|}
\hline Thème & Année & Directeur(s) du bulletin \\
\hline Tout un programme & 2002 & Nelly Labère \\
\hline Paysages de la mémoire & 2002 & Nelly Labère \\
\hline Les bruits de la ville & 2003 & Estelle Doudet et Andrea Martignoni \\
\hline L'arbre & 2003 & Nelly Labère \\
\hline Autour de la mort & 2003 & Estelle Doudet \\
\hline La nuit & 2004 & Nelly Labère et Andrea Martignoni \\
\hline Les tabous & 2004 & Nelly Labère \\
\hline Figures de l'autorité & 2006 & Julien Abed et Sophie Albert \\
\hline À la marge & 2006 & Julien Abed et Sophie Albert \\
\hline Croire & 2006 & Julien Abed, Andrea Martignoni et Sophie Albert \\
\hline La transmission & 2007 & Sophie Albert \\
\hline La faim et l'appétit & 2007 & Anne-Laure Lalouette \\
\hline
\end{tabular}




\begin{tabular}{|c|c|c|}
\hline $\begin{array}{l}\text { Figures royales à l'ombre du } \\
\text { mythe }\end{array}$ & 2008 & Natalia Bercea-Bocksai et Amandine Mussou \\
\hline Violences médiévales & 2008 & Léonard Dauphant \\
\hline Les activités quotidiennes & 2008 & Irène Fabry-Tehranchi et Cécile Le Cornec \\
\hline Secret, public, privé & 2009 & Clémence Revest \\
\hline Les hommes illustres & 2009 & Marion Chaigne-Legouy et Anne Salamon \\
\hline La règle du jeu & 2010 & Amandine Mussou et Laëtitia Tabard \\
\hline Les manifestations divines & 2010 & Isabelle Coumert et Céline Ménager \\
\hline Maris et femmes & 2010 & $\begin{array}{l}\text { Diane Chamboduc de Saint-Pulgent et Blandine } \\
\text { Longhi }\end{array}$ \\
\hline $\begin{array}{l}\text { Grivoiserie, pornographie, } \\
\text { scatologie }\end{array}$ & 2011 & Mathilde Grodet et Marie Dupuy \\
\hline La route des pèlerins & 2011 & Magali Cheynet \\
\hline Le doute & 2012 & Servane Michel et Francesco Montorsi \\
\hline L'identité & 2012 & $\begin{array}{l}\text { Cécile Becchia, Diane Chamboduc de Saint-Pulgent } \\
\text { et Fanny Oudin }\end{array}$ \\
\hline L'habit fait-il le moine? & 2013 & Fanny Oudin \\
\hline Trouver la paix & 2013 & Stéphanie Richard et Irène Strobbe \\
\hline Naissances & 2014 & Emilie Deschelettes et Céline Ménager \\
\hline La prophétie & 2014 & Servane Rayne-Michel et Maria Valeria Ingegno \\
\hline Copie, authenticité, originalité & 2015 & $\begin{array}{l}\text { Jean-Baptiste Camps, Magali Cheynet et Vincent Le } \\
\text { Quentrec }\end{array}$ \\
\hline L'erreur, l'échec, la faute & 2015 & Florian Besson et Catherine Kikuchi \\
\hline Le temps de la fête & 2015 & Sarah Delale et Jean-Dominique Delle Luche \\
\hline Faire communauté & 2016 & Julia Conesa et Julie Pilorget \\
\hline Finir le Moyen Âge & 2016 & Pauline Guéna et Annabelle Marin \\
\hline L'hiver au Moyen Âge & à & Anne Kucab et Elodie Pinel \\
\hline Cultures de l'autre & pà & Pauline Guéna et Viviane Griveau-Genest \\
\hline
\end{tabular}




\begin{tabular}{|l|l|l|}
\hline $\begin{array}{l}\text { Faire de l'histoire au Moyen } \\
\text { Âge }\end{array}$ & $\begin{array}{l}\text { à } \\
\text { paraître }\end{array}$ & $\begin{array}{l}\text { Pauline Bouchaud, Mélanie Fougre-Lévêque et } \\
\text { François Wallerich }\end{array}$ \\
\hline Sexualités et interdits & $\begin{array}{l}\text { à } \\
\text { paraître }\end{array}$ & Catherine Kikuchi et Elodie Guilhem \\
\hline
\end{tabular}

Outre ces thèmes, Questes a également organisé sept journées d'études, tous les deux ans depuis 2003 : les quatre premières ont été publiées aux Presses de Paris-Sorbonne, sous les couvertures et affiches suivantes.

Les couvertures des journées d'études de Questes

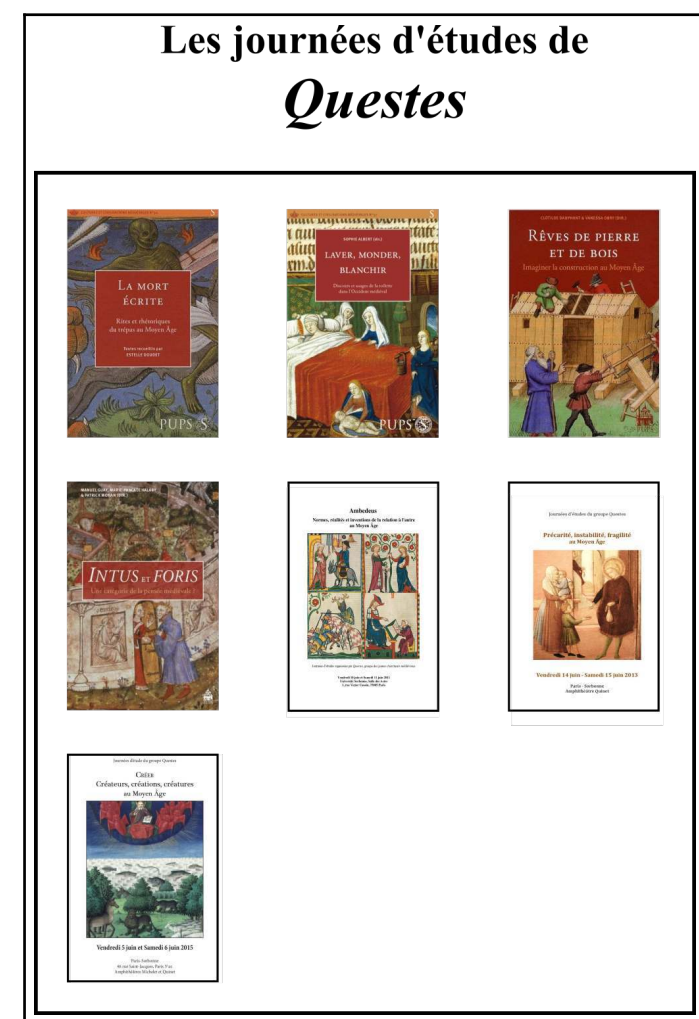

À la lecture de ces trente-sept thèmes, plusieurs conclusions s'imposent. On remarque d'abord que de nombreux thèmes sont étroitement liés à l'actualité historiographique : en 2004, le numéro 6 se penche sur La nuit au Moyen Âge, quelques mois après la republication du livre de Jean Verdon ${ }^{16}$ ou d'un numéro dédié à ce thème dans la Revue des langues romanes $^{17}$. Daté de 2013, le numéro 26 , Trouver la paix, peut être rapproché de plusieurs ouvrages qui sortent dans un intervalle de temps relativement rapproché ${ }^{18}$. Plus proche encore, le numéro 33, Finir le Moyen Âge, répond à la publication de l'ouvrage de Jacques Le Goff sur les périodisations en histoire ${ }^{19}$, sorti quelques semaines plus tôt. D'autres, à l'inverse, anticipent certaines tendances de la recherche : en 2003, le numéro 3 est ainsi consacré aux Bruits de la ville, treize ans avant la publication d'un ouvrage sur les paysages sonores médiévaux ${ }^{20}$. Un grand nombre de thèmes ont des résonances familières plus diffuses pour des médiévistes car ils recoupent de grandes tendances qui structurent la recherche du moment: ainsi du numéro 5, Autour de la mort, qui témoigne de l'intérêt 
pour ces thèmes dans les années 1990 , ou du futur numéro 35 , Cultures de l'autre, qui reprend les questionnements actuels de l'histoire globale ou connectée. Les convergences entre les activités de Questes et l'actualité de la recherche sont dans ce dernier cas tout à fait notables: en mai 2016, le congrès de la Société des Historiens Médiévistes de l'Enseignement Supérieur Public (SHMESP) porte significativement sur l'histoire-monde et les espaces connectés.

Si on essaie de synthétiser ces thèmes, quatre grandes tendances semblent se dégager, les Questeurs se concentrant surtout sur :

La question des normes, à la fois sociales, morales et imaginaires, et celle, corrélative, des transgressions : numéros 7 (Les tabous), 8 (Figures de l'autorité), 9 (À la marge), 14 (Violences médiévales), 16 (Secret, public, privé), 18 (La règle du jeu), 21 (Grivoiserie, pornographie, scatologie), 30 (L'erreur, l'échec, la faute), futurs numéros 34 (Cultures de l'autre) et 36 ( Sexualités et interdits). On pourrait également placer dans cette tendance la journée d'études de 2011, Ambedeus. Normes, réalités et inventions de la relation à l'autre.

Le spirituel, au sens large, et les pratiques sociales qui en découlent - citons les numéros 5 (Autour de la mort), 10 (Croire), 11 (La transmission), 19 (Les manifestations divines), 22 (La route des pèlerins), 23 (Le doute), 26 (Trouver la paix) et 28 (La prophétie). Il faut y ajouter la journée d'études de 2003 (La mort écrite), de 2009 (Intus et foris) et de 2015 (Créer. Créateurs, créations, créatures).

Les pratiques sociales et la vie quotidienne : numéros 3 (Les bruits de la ville), 12 (La faim et l'appétit), 15 (Les activités quotidiennes), 20 (Maris et femmes), 25 (L'habit fait-il le moine ?), 27 ( Naissances), 31 (Le temps de la fête), 33 (L'hiver). À quoi s'ajoutent les journées d'études de 2005 (Laver, monder, blanchir), 2007 (Imaginer la construction au Moyen Âge), 2013 ( Précarité, instabilité, fragilité), et, dans une certaine mesure, de 2015 (Créer. Créateurs, créations, créatures).

Enfin, depuis quelque temps, des thèmes que l'on pourrait appeler « méta-réflexifs », qui se penchent sur les sources elles-mêmes, sur des questions de périodisation, ou sur les historiens médiévaux : les numéros 29 (Copie, authenticité, originalité), 33 (Finir le Moyen Âge ) et 35 (Faire de l'histoire au Moyen Âge).

29 Là aussi, la pluridisciplinarité a des conséquences très concrètes : certains champs de recherche importants pour les historiens, comme le territoire, le pouvoir, ou l'histoire économique au sens large, brillent par leur absence, les thèmes devant être à chaque fois à même d'inspirer toutes les disciplines. De même, les problématiques purement littéraires, linguistiques et philologiques n'interviennent qu'à travers des catégories plus thématiques, comme le montre admirablement le numéro 29 (Copie, authenticité, originalité ).

\section{Des articles}

Ces trente-trois numéros rassemblent, sans compter les avant-propos rédigés par des professeurs ni les introductions, conclusions et bibliographies rédigées par les coordinateurs des numéros, cent-quatre-vingt-deux articles. Certains numéros comprennent jusqu'à sept ou huit articles, d'autres sont plus légers (numéro 5 , Autour de la mort). Les premiers numéros présentent souvent des comptes rendus ou des notes de lecture: ce format disparaît assez vite et mériterait probablement d'être réinvesti. De même, les premiers numéros proposent de brèves notes sur des sites internet utiles à la 
médiévistique contemporaine; ce format, baptisé "L'Esplumoir virtuel», disparaît en 2004, probablement avec le départ de son auteur, Aimeric Vacher. Cette catégorie mérite une brève mention car elle témoigne d'une grande clairvoyance de la part de son auteur: en 2003-2004, quand Internet n'en était encore qu'à ses débuts, les articles d'Aimeric Vacher accompagnaient les années décisives de l'implantation du Web: entre 2001 et 2005, la proportion de foyers français ayant Internet passe significativement de $17 \%$ à $45 \%$. Depuis cette époque, Internet a évidemment beaucoup changé : certains sites mentionnés dans «L'Esplumoir virtuel » n'existent plus aujourd'hui - ainsi de la Guilde des Doctorants -, mais d'autres sont toujours des références, comme The Chronicle of Higher Education.

Pour l'instant, tous ces articles sont en français: peut-être l'avenir permettra-t-il à Questes d'accueillir des articles rédigés dans d'autres langues. La longueur des articles varie significativement : si les premiers articles ne font parfois que quelques paragraphes, d'autres, en particulier dans les derniers numéros, atteignent jusqu'à vingt, voire trente pages. En tout, cela représente plusieurs milliers de pages. Ce corpus a permis la réalisation d'un nuage de mots (wordcloud) présenté ci-dessous. Nous en avons ôté les verbes, les adjectifs, les adverbes, pour ne garder que les noms communs ou des noms propres fréquents, comme Arthur ou Merlin. Les résultats sont éclairants et offrent une belle image de l'activité de Questes sur ces quinze dernières années.

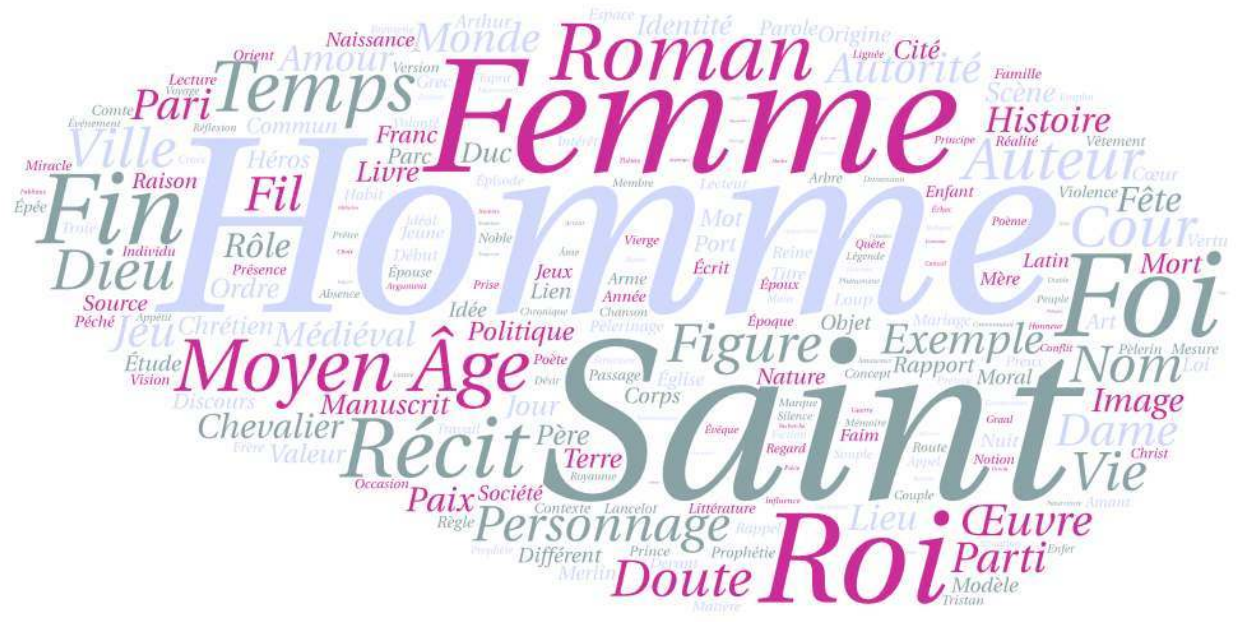

32 À ces articles, il faut ajouter les avant-propos, où l'on rencontre les noms de chercheurs renommés qui nous ont fait l'honneur d'écrire pour Questes: Dominique Barthélémy, Danièle Alexandre-Bidon, Jean-Michel Mehl, Didier Lett, Cécile Caby, Bernard Ribemont, Joseph Morsel, Catherine Vincent, et d'autres encore. Il ne faut également pas oublier tous ceux qui nous ont offert et nous offrent encore leur protection dans les instances administratives de l'université. C'est aussi grâce à leur soutien et à leur bienveillance que Questes peut continuer à exister, et cet article anniversaire est l'occasion de redire à tous notre reconnaissance.

Que dire pour finir? Peut-être, encore une fois, faut-il se tourner vers les mots. Sur revues.org, les bulletins déclinent près de huit cents mots-clés, de « Abélard » à «Ysaie le Triste ", ce qui reflète à la fois l'étendue des thèmes abordés et la forte pluridisciplinarité des numéros. Parmi les plus fréquents figurent "femme", "chevalerie», «jeu», "mélancolie", «filiation", "voyage»: tous les Questeurs d'aujourd'hui et d'hier y retrouveront sans aucun doute au moins un de leurs intérêts de recherche. Plus fréquemment encore, on rencontre « identité », « mort », « mythe » et « violence » ${ }^{21}$; ou, 
à égalité, et tâchons de ne pas y voir là encore le simple reflet des préoccupations des doctorants, « sexualité » et « alimentation »...

Le mot-clé le plus fréquent, et c'est un beau symbole, est "mémoire ", suivi de très près par « transmission » : comment mieux dire ce qui nous lie et nous anime?

Nous rappelions que Questes s'était construit sous le signe de l'internationalité, de l'interdisciplinarité et de la convivialité. À l'image des Trois Mousquetaires, ce triplé masque un membre caché : l'amitié. C'est donc à un court florilège effeuillant cette belle vivace que les Questeurs, s'étant fait jardiniers, vous invitent en conclusion :

Au besoing, toz jorz le dit en, / Doit on son ami esprouver ${ }^{22}$.

Il y avait dans l'armée du roi Foulques fils de Foulques un chevalier franc respectable, qui était venu de leurs contrées pour accomplir le pèlerinage et s'en retourner ensuite. Il devint mon familier et s'attacha à moi au point qu'il m'appelait : mon frère. L'amitié avait créé entre nous des relations suivies ${ }^{23}$.

Len ne puet avoir trop d'amis ${ }^{24}$.

Vous qui êtes ma mère par votre fonction et ma sœur bien-aimée par l'amitié, vous que j'honore pour la piété, la foi, l'affection, le cœur, par sentiment céleste, sans ombre d'implication du corps, en vous j'aime non pas ce que désire la chair, mais l'esprit ${ }^{25}$.

Godefroy de Bouillon et Hugues de France... chacun de ces deux était pour l'autre comme un second lui-même, car leur amitié était pareille ${ }^{26}$.

Longe demoree fait ami noval ${ }^{27}$.

Car Amours dist que au grant besoing voit li homs son amy, or me respons cy : se je ne li aide adont, quant li aiderai jou ? Di moi, par amours, quant li aideray jou ${ }^{28}$ ?

Mieux vault amis en voie que deniers en corroie ${ }^{29}$.

Cellui est vray ami qui t'aime quant tous les autres te faillent ${ }^{30}$.

\section{NOTES}

1. Estelle Doudet, entretien avec Élodie Pinel. Sur la fondation du groupe et ses activités durant l'année scolaire 2001-2002, on pourra consulter les extraits du premier numéro de la revue, Tout un programme, Questes [en ligne], $\mathrm{n}^{\mathrm{o}} 1,2002$, mis en ligne le $1^{\mathrm{er}}$ janvier 2014, consulté le 19 mai 2016. URL: http://questes.revues.org/247.

2. Elle en compte aujourd'hui quatre cent soixante dix-sept.

3. Les envois des premiers bulletins papiers ont même été, dans les premiers temps, assumés sur les fonds propres de Nelly Labère.

4. Tous les numéros ont été transférés sur revues.org, où l'on observera aisément cette évolution du format de la revue $:$ http://questes.revues.org.

5. Les citations suivantes sont tirées des réponses reçues à un questionnaire soumis par les auteurs des présentes lignes aux anciens Questeurs.

6. Ibid.

7. Ibid.

8. Ibid.

9. Ibid.

10. Ibid. 
11. Questes, «Questes passées : l'activité du groupe en 2001-2002 », Questes [en ligne], $\mathrm{n}^{\circ} 1,2002$, mis en ligne le $1^{\mathrm{er}}$ janvier 2014, consulté le 19 mai 2016. URL : http://questes.revues.org/3589.

12. Les communications sont parues sous la direction de Natalia Bercea-Bocskai et Amandine Mussou dans Figures royales à l'ombre du mythe: Questes [en ligne], $\mathrm{n}^{\circ} 13,2008$, mis en ligne le $1^{\mathrm{er}}$ janvier 2014, consulté le 19 mai 2016. URL : http://questes.revues.org/228.

13. Il s'agit des numéros numéros 3, 6, 10, 17, 20, 24, 27, 29 et 31 .

14. Parmi les Questeurs les plus actifs dans les premières années, on trouve en effet de très nombreux doctorants de Jacqueline Cerquiglini-Toulet, notamment Julien Abed, Sophie Albert, Mattia Cavagna, Clotilde Dauphant, Estelle Doudet, Midoriko Kageyama, Nelly Labère, Pierre Levron, Amandine Mussou, Fanny Oudin, Agathe Sultan et Laëtitia Tabard.

15. Dans le conseil d'administration des années 2014-2016, on rencontre en effet Florian Besson, Adrien Carbonnet, Diane Chamboduc de Saint-Pulgent, Pauline Guéna, Anne Kucab, Catherine Kikuchi, Annabelle Marin, Julie Pilorget, Stéphanie Richard et Cécile Troadec, tous doctorants sous la direction d'Élisabeth Crouzet-Pavan.

16. Jean Verdon, La Nuit au Moyen Âge, Paris, Hachette, 2003 [1994].

17. Revue des langues romanes, «L'imaginaire de la nuit au Moyen Âge », 106, nº 2, 2002.

18. Jenny Benham, Peacemaking in the Middle Ages : principles and practice, Manchester/New York, Manchester University Press, 2011; Faire la guerre, faire la paix : approches sémantiques et ambiguïtés terminologiques, dir. Isabelle Chave, Paris, Comité des travaux historiques et scientifiques, édition électronique, 2012 ; Faire la paix et se défendre à la fin du Moyen Âge, dir. Gisela Naegle, Oldenbourg Verlag, coll. «Pariser historische Studien», 2012 ; Jenny Benham, Peacemaking in the Middle Ages: principles and practice, Manchester/New York, Manchester University Press, 2011.

19. Jacques Le Goff, Faut-il vraiment découper l'histoire en tranches ?, Paris, Éditions du Seuil, 2014.

20. Les Paysages sonores du Moyen Âge à la Renaissance, dir. Laurent Hablot et Laurent Vissière, Rennes, PUR, 2016.

21. Notons que ces résultats sont quelque peu biaisés : «femme », « identité » et « violence » sont très représentés car ils ont fait l'objet d'un numéro à part entière, respectivement les numéros 20, Maris et femmes, 24, L'identité, et 14, Violences médiévales.

22. Chrétien de Troyes, Le Chevalier au lion ou le Roman d'Yvain, éd. David F. Hult, Paris, Livre de poche, coll. « Lettres gothiques », 2004, v. 6590-6591, p. 456.

23. Usāma ibn Munqidh, Des Enseignements de la vie. Souvenirs d'un gentilhomme syrien du temps des Croisades, trad. André Miquel, Paris, Imprimerie Nationale, 1983, p. 291.

24. Proverbes français antérieurs au $X V^{e}$ siècle, éd. Joseph Morawski, Paris, Champion, 2007 [1925], proverbe 1508.

25. Venance Fortuna à des moniales, Poèmes, trad. M. Reydellet, Paris, Les Belles Lettres, 2002, vol. 3, XI, VI, p. 116.

26. Robert le Moine, "Historia Iherosolimitana", dans Recueil des Historiens des Croisades, Historiens Occidentaux, Paris, Imprimerie impériale, 1866, t. III, VII, 11, p. 831.

27. Proverbes français antérieurs au $X V^{e}$ siècle, éd cit., proverbe 1140.

28. Marguerite Porete, Le Mirouer des simples ames, ch. 77, Valenciennes, Bibliothèque municipale, 0239 (230), fo $69 \mathrm{v}^{\circ}$.

29. Proverbes français antérieurs au $X V^{e}$ siècle, éd. cit., proverbe 1241.

30. Disciplina clericalis; auctore Petro Alphonsi, ex-judceo hispano. Pars prima. = Discipline de clergie; traduction de l'ouvrage de Pierre Alphonse. Première partie, éd. J. Labouderie, Paris, Rignoux pour la Société des bibliophiles français, 1824, p. 15. 


\section{AUTEURS}

FLORIAN BESSON

Université Paris-Sorbonne

\section{ADRIEN CARBONNET}

Université Paris-Sorbonne

SARAH DELALE

Université Paris-Sorbonne

\section{PAULINE GUENA}

Université Paris-Sorbonne

\section{ELODIE PINEL}

Université Paris Ouest Nanterre La Défense 